\title{
The blooming of the French lilac
}

\author{
Lee A. Witters \\ Endocrine-Metabolism Division, Departments of Medicine and Biochemistry, \\ Dartmouth Medical School, Remsen 322, Hanover, New Hampshire 03755-3833, USA. \\ Phone: (603) 650-1909; Fax: (603) 650-1727; E-mail: lee.a.witters@dartmouth.edu.
}

J. Clin. Invest. 108:1105-1107 (2001). DOI:10.1172/JCI200114178.

The history of diabetes mellitus is replete with many therapies, nearly all, including insulin, first given without any knowledge of a mechanism of action. Beginning with early Egyptian physicians who, according to the Ebers Papyrus of 1500 B.C.E., prescribed a mixture of cakes, wheat grains, fresh grits, green lead, earth, and water, up to the more recent introduction of the thiazolidinediones, knowledge of physiologic effects preceded by years or centuries any knowledge as to what the medicament actually did.

In medieval times, a prescription of Galega officinalis was said to relieve the intense urination accompanying the disease that came to have the name of diabetes mellitus. G. officinalis, also known as Goat's rue, the French lilac or Italian fitch (Figure 1), was also given during the plague epidemics to promote perspiration and has been used as a galactogogue in cows. The active ingredient in the French lilac that produced the lowering of blood glucose was shown to be galegine or isoamylene guanidine (1). A curious chapter in the history of guanidinebased hypoglycemic agents arose from the mistaken notion that the tetany of hypoparathyroidism was due to the production of increased guanidine following parathyroidectomy, leading to the demonstration that an infusion of guanidine produced lowering of blood glucose (2). While guanidine itself and certain derivatives are too toxic for the treatment of diabetes mellitus, the biguanides (two linked guanidine rings) have proved useful, and three biguanides became available for diabetes therapy in the 1950s. Phen-

\section{Figure 1}

The bloom of the French lilac. This blooming $G$. officinalis (Goat's rue; French lilac; Italian fitch) is rich in guanidine. The plant's long-recognized hypoglycemic properties led eventually to the synthesis of the biguanide compound metformin. formin and buformin, the former becoming quite popular in the $1960 \mathrm{~s}$, were withdrawn from the pharmacopoeia in the early 1970s due to the emergence of frequent lactic acidosis and increased cardiac mortality (1). Metformin, a less lipophilic biguanide, proved safer and, after 20 years of use in Europe, was approved for use in the USA in 1995.

\section{Metformin in the clinic}

Metformin is now a mainstay of therapy in the treatment of type 2 diabetes and, as noted in the recently terminated Diabetes Prevention Program, is also an effective agent to decrease the risk of development of the disease (1, $3,4)$. In the United Kingdom Prospective Diabetes Study, metformin also proved to be effective in decreasing diabetes-related death, myocardial infarction, and stroke (5). Metformin not only lowers blood glucose but also inhibits adipose tissue lipolysis, reduces circulating free fatty acids, and diminishes VLDL production $(1,3,4)$. Among the known actions of metformin are an improvement in insulin sensitivity in muscle and liver, a decrease in hepatic glucose production via gluconeogenesis, an increase in

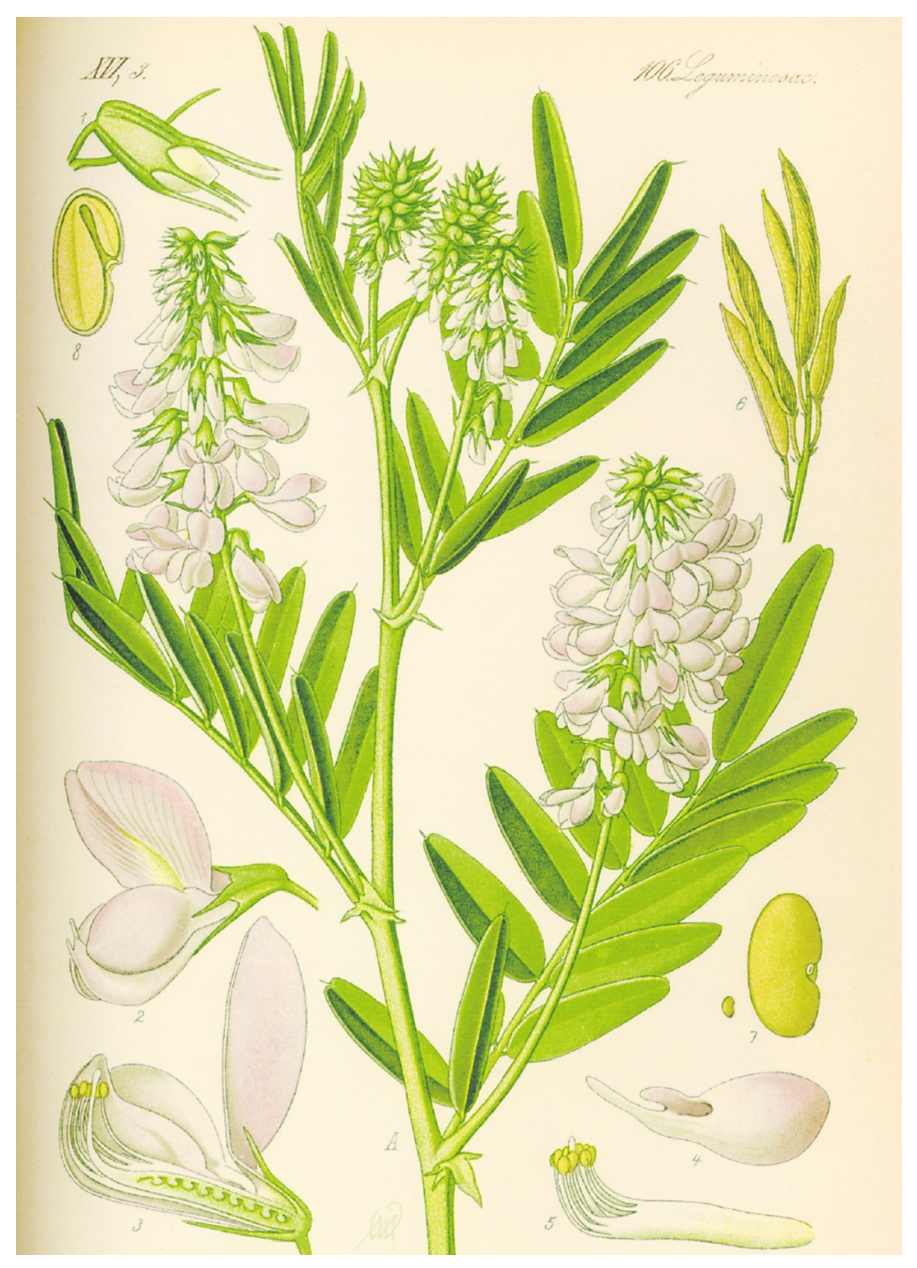


peripheral glucose utilization (predominantly through a stimulation of insulin-mediated muscle glucose uptake and glycogen synthesis), and positive effects on insulin receptor expression and tyrosine kinase activity (1). In addition, metformin appears to suppress the gluconeogenic effects of glucagon and to increase the translocation of glucose transporters to the cell surface (4). The exact molecular locus of metformin's effects, however, remain obscure. Two recent studies suggested that its target, albeit indirect, is the respiratory chain complex I, leading to an inhibition of mitochondrial respiration $(6,7)$.

\section{Metformin and the AMP- activated protein kinase}

In this issue of the JCI, Zhou and coworkers provide evidence that the elusive target of metformin's actions is the AMP-activated protein kinase (AMPK) (8). In studies performed in isolated hepatocytes and rat skeletal muscles, they demonstrate that metformin leads to AMPK activation, accompanied by an inhibition of lipogenesis (due to inactivation of acetylCoA carboxylase and suppression of lipogenic enzyme expression), suppression of the expression of SREBP-1 (a central lipogenic transcription factor), and a modest stimulation of skeletal muscle glucose uptake. Similar hepatic effects are seen in metformin-treated rats. Based on the use of a newly discovered AMPK inhibitor, their data suggest that the ability of metformin to suppress glucose production in hepatocytes requires AMPK activation. Many of these effects are similar to that of 5-aminoimidazole-4-carboxamide ribonucleoside (AICAR), a known, albeit nonspecific, activator of AMPK (9). However, metformin does not lead to AMPK activation in vitro, indicating that it activates the kinase indirectly. Thus, the tale of metformin's proximal cellular target continues.

AMPK, first identified as a kinase active on HMG-CoA reductase, is one important member of a large serine/threonine protein kinase family, called the AMPK/SNF1 family (10-12). This family is found in all eukaryotes, including plants, yeast, Drosophila, Caenorhabditis elegans, and mammals. AMPK is a heterotrimeric enzyme, consisting of an $\alpha$ catalytic subunit and noncatalytic, regulatory $\beta$ and $\gamma$ sub- units. Each AMPK subunit is represented in an isoform family, derived from different genes. The binding of the $\beta / \gamma$ subunits to the $\alpha$ subunit regulates enzyme activity, targeting, and $\alpha$ protein turnover (13). Both the $\alpha$ (multisite phosphorylation via upstream AMPKKs) and $\beta$ (myristoylation, multisite phosphorylation) subunits are regulated by posttranslational modification $(10-12,14)$. The $\gamma$ subunits, while not posttranslationally modified, are composed of repetitive CBS domains (for cystathione $\beta$-synthase where they were first identified) and are critical for AMP regulation $(15,16)$.

AMPK subunits appear to be expressed in all mammalian tissues with a mixture of isoforms and specific heterotrimers represented in a tissuespecific fashion. AMPK is subject to complex regulation via allosteric and covalent mechanisms, which alter catalytic subunit activity, its heterotrimer structure, and its cellular localization (10-12). Allosteric regulation by AMP and ATP was the first recognized regulatory mechanism. The enzyme is very sensitive to AMP activation $\left(\mathrm{K}_{\mathrm{a}} 20 \mu \mathrm{M}\right)$ and is inhibited at high ATP. There is no consensus AMP binding site on any of the subunits; AMP allostery appears to be due to a higher-order structure, involving all the subunits, but especially the $\gamma$ subunit. This sensitivity to AMP and ATP creates a metabolic sensor that is able to monitor very small changes in cellular ATP content. During early ATP depletion, as in limited glucose availability, glycogen exhaustion, muscular exercise, or hypoxia, adenylate kinase catalyzes the resynthesis of ATP from accumulating ADP $(2 \mathrm{ADP} \rightarrow \mathrm{ATP}+\mathrm{AMP})$. This means that the ratio of AMP to ATP actually varies as a function of (ADP/ATP $)^{2}$, making AMPK very sensitive to minimal ATP depletion. While Zhou et al. report no changes in cellular ATP levels in their study, no measurements of AMP are given. Subtle but significant changes in cellular adenine nucleotide pools, perhaps as a function of metformin's ability to inhibit cellular respiration, might account for its AMPK-activating effects. Creatine phosphate, which accumulates in skeletal muscle only when a surfeit of ATP exists, also serves as a negative allosteric regulator. ZMP (the monophosphorylated derivative of AICAR) mimics the actions of AMP to activate AMPK; AICAR, like met- formin, has been shown to decrease hepatic glucose production and gluconeogenesis, to inhibit lipolysis, lipogenesis, and lipogenic gene expression, and to increase skeletal muscle glucose uptake $(9,17-19)$.

\section{Targeting AMPK}

Is AMPK a promising target for the treatment of diabetes mellitus? Its known protein and cellular targets certainly suggest so (20). Several studies, with varying degrees of experimental rigor, point to its important roles in the suppression of gluconeogenesis in the liver, promotion of glucose uptake in skeletal muscle, inhibition of fatty acid and sterol synthesis, increases in fatty acid metabolism through oxidation, and inhibition of lipolysis $(17-19,21)$. AMPK also phosphorylates and activates endothelial nitric oxide synthase (eNOS), which could have positive effects on capillary blood flow important for the diabetic (22). Some caution is appropriate, however, for other actions of an activated AMPK could produce deleterious effects. AMPK is known to interact with and inhibit the cystic fibrosis transmembrane conductance regulator (CFTR) (23). Under certain conditions, AMPK activation may lead to an inhibition of insulin secretion (24). The promotion of fatty acid oxidation (through its ability to phosphorylate and inhibit acetyl-CoA carboxylase, lowering malonyl-CoA) could decrease cardiac function and efficiency after ischemia during reperfusion (25). Recently, a mutation in the gene encoding AMPK's $\gamma$ - 2 subunit has been pinpointed as a molecular defect underlying ventricular pre-excitation in the Wolff-Parkinson-White syndrome (26). This mutation, which maps to the first CBS domain of the gamma subunit, leads to an AMP-independent activation of AMPK activity (15); thus, heightened AMPK activity could lead to developmental and/or functional aberrancy of cardiac conduction. AMPK activation could also have undesirable effects on cellular differentiation, not limited to its known effects on adipogenesis (27).

Nonetheless, the emerging central role of AMPK in the regulation of carbohydrate and lipid metabolism provides a compelling story, heightened by the current report of Zhou and coworkers on metformin (8). The bloom of the French lilac may be real- 
ized in the flowering of a new direction in the therapy of diabetes mellitus.

\section{Acknowledgments}

The author would like to acknowledge the support of NIH grant DK-35712.

1. Cusi, K., and Defronzo, R.A. 1998. Metformin: a review of its metabolic effects. Diabetes Reviews. 6:89-131.

2. Watanabe, C.K. 1918. Studies in the metabolic changes induced by administration of guanidine bases. I. Influence of injected guanidine hydrochloride upon blood sugar content. J. Biol. Chem. 33:253-265.

3. Bailey, C.J., and Turner, R.C. 1996. Metformin. N. Engl. J. Med. 334:574-579.

4. Wiernsperger, N.F., and Bailey, C.J. 1999. The antihyperglycemic effect of metformin: therapeutic and cellular mechanisms. Drugs. 58:31-39.

5. UKPDS Group. 1998. Effects of intensive bloodglucose control with metformin on complications in overweight patients with type 2 diabetes. Lancet. 352:854-865.

6. El-Mir, M.-Y., et al. 2000. Dimethylbiguanide inhibits cell respiration via an indirect effect targeted on the respiratory chain complex I. J. Biol. Chem. 275:223-228.

7. Owen, M.R., Doran, E., and Halestrap, A.P. 2000 Evidence that metformin exerts its anti-diabetic effects through inhibition of complex 1 of the mitochondrial respiratory chain. Biochem. J. 348:607-614.

8. Zhou, G., et al. 2001. Role of AMP-activated protein kinase in mechanism of metformin action. $J$ Clin. Invest. 108:1167-1174.
9. Corton, J.M., Gillespie, J.G., Hawley, S.A., and Hardie, D.G. 1995. 5-aminoimidazole-4-carboxamide ribonucleoside. A specific method for activating AMP-activated protein kinase in intact cells? Eur. J. Biochem. 229:558-565.

10. Kemp, B.E., et al. 1999. Dealing with energy demand: the AMP-activated protein kinase. Trends Biochem. Sci. 24:22-25.

11. Hardie, D.G., Carling, D., and Carlson, M. 1998. The AMP-activated/SNF1 protein kinase subfamily: metabolic sensors of the eukaryotic cell? Annu. Rev. Biochem. 67:821-855.

12. Hardie, D.G., and Carling, D. 1997. The AMPactivated protein kinase: fuel gauge of the mammalian cell? Eur. J. Biochem. 246:259-273.

13. Crute, B.E., Seefeld, K., Gamble, J., Kemp, B.E., and Witters, L.A. 1998. Functional domains of the alpha1 catalytic subunit of the AMP-activated protein kinase. J. Biol. Chem. 273:35347-35354.

14. Warden, S.M., et al. 2001. Post-translational modifications of the beta- 1 subunit of AMP-activated protein kinase affect enzyme activity and cellular localization. Biochem. J. 354:275-283.

15. Hamilton, S.R., et al. 2001. An activating mutation in the gamma-1 subunit of the AMP-activated protein kinase, FEBS Lett. 500:163-168.

16. Cheung, P.C., Salt, I.P., Davies, S.P., Hardie, D.G. and Carling, D. 2000. Characterization of AMPactivated protein kinase gamma-subunit isoforms and their role in AMP binding. Biochem. J. 346:659-669.

17. Lochhead, P.A., Salt, I.P., Walker, K.S., Hardie D.G., and Sutherland, C. 2000. 5-aminoimidazole-4-carboxamide riboside mimics the effects of insulin on the expression of the 2 key gluconeogenic genes PEPCK and glucose-6-phosphatase. Diabetes. 49:896-903.

18. Hayashi, T., et al. 2000. Metabolic stress and altered glucose transport: activation of AMP-acti- vated protein kinase as a unifying coupling mechanism. Diabetes. 49:527-531.

19. Bergeron, R., et al. 2001. Effect of 5-aminoimidazole-4-carboxamide-1-beta-D-ribofuranoside infusion on in vivo glucose and lipid metabolism in lean and obese Zucker rats. Diabetes. 50:1076-1082.

20. Winder, W.W., and Hardie, D.G. 1999. AMP-activated protein kinase, a metabolic master switch: possible roles in type 2 diabetes. Am. J. Physiol. 277:E1-E10.

21. Mu, J., Brozinick, J.T., Jr., Valladares, O., Bucan, M., and Birnbaum, M.J. 2001. A role for AMPactivated protein kinase in contraction- and hypoxia-regulated glucose transport in skeletal muscle. Mol. Cell. 7:1085-1094.

22. Chen, Z.P., et al. 1999. AMP-activated protein kinase phosphorylation of endothelial NO syn thase. FEBS Lett. 443:285-289.

23. Hallows, K.R., Raghuram, V., Kemp, B.E., Witters, L.A., and Foskett, J.K. 2000. Inhibition of cystic fibrosis transmembrane conductance regulator by novel interaction with the metabolic sensor AMP-activated protein kinase. J. Clin. Invest. 105:1711-1721.

24. Salt, I.P., Johnson, G., Ashcroft, S.J., and Hardie, D.G. 1998. AMP-activated protein kinase is activated by low glucose in cell lines derived from pancreatic beta cells, and may regulate insulin release. Biochem. J. 335:533-539.

25. Lopaschuk, G.D. 1997. Alterations in fatty acid oxidation during reperfusion of the heart afte myocardial ischemia. Am. J. Cardiol. 80:11A-16A.

26. Gollob, M.H., et al. 2001. Identification of a gene responsible for a familial Wolff-Parkinson-White syndrome. N. Engl. J. Med. 344:1823-1831.

27. Habinowski, S., and Witters, L.A. 2001. The effects of AICAR on adipocyte differentiation of 3T3-L1 cells. Biochem. Biophys. Res. Commun. 286:852-856. 